\title{
Viscoelastic Properties of Bronchial Mucus After Respiratory Physiotherapy in Subjects With Bronchiectasis
}

\author{
Ercy MC Ramos PhD, Dionei Ramos PhD, Graciane L Moreira PhD, Mariangela Macchione PhD, \\ Eliane T Guimarães PhD, Fernanda Maria M Rodrigues MSc, Altay Alves Lino de Souza PhD, \\ Paulo HN Saldiva PhD, and José R Jardim PhD
}

\begin{abstract}
BACKGROUND: Previous studies have evaluated the effectiveness of postural drainage (PD), percussion (PERC), the coughing technique (CT), and other types of coughing in subjects with bronchiectasis. However, the application times of these techniques and the quality of the expectorated mucus require further study. The aim of our study was to evaluate the effectiveness of PD, percussion, CT, and huffing in subjects with bronchiectasis and assess the quantity and quality of bronchial mucus produced (measurement of wet and dry weight and determination of viscoelastic properties). METHODS: Twenty-two subjects with stable bronchiectasis ( 6 men; mean age: 51.5 y) underwent $4 \mathrm{~d}$ of experimental study (CT, PD+CT, PD+PERC+CT, and PD+huffing). The techniques were performed in 320 -min periods separated by 10 min of rest. Before performing any technique (baseline) and after each period $(30,60$, and $90 \mathrm{~min})$, expectorated mucus was collected for analysis of viscoelasticity. RESULTS: A significant increase in the dry weight/wet weight ratio was found after $60 \mathrm{~min}$ of PD+PERC $+\mathrm{CT}(P=.01)$ and $90 \mathrm{~min}$ of $P D+h u f f i n g(P=.03)$ and PD+PERC+CT $(P=.007)$ in comparison with CT. PD+PERC+CT and PD+huffing led to the greatest removal of viscoelastic mucus at $60 \mathrm{~min}(P=.02$ and $P=.002$, respectively) and continued to do so at $90 \mathrm{~min}(P=.02$ and $P=.01$, respectively $)$ in comparison with $C T$. An interaction effect was found, as all techniques led to a greater removal of elastic mucus in comparison with CT at 60 min $(P D+C T, P=.001 ; P D+P E R C+C T, P<.001 ; P D+h u f f i n g, P<.001)$, but only $\mathrm{PD}+\mathrm{PERC}+\mathrm{CT}$ and PD+huffing led to a greater removal of elastic mucus than $\mathrm{CT}$ at $90 \mathrm{~min}$ $(P<.001$ and $P=.005$, respectively). CONCLUSIONS: PD+PERC+CT and PD+huffing performed similarly regarding the removal of viscoelastic mucus in 2 and 320 -min periods separated by $10 \mathrm{~min}$ of rest. PD+PERC+CT led to the greatest removal of mucus in the shortest period ( 2 20-min periods separated by 10 min of rest). Key words: bronchiectasis; mucus; viscosity; elasticity; cough; physical therapy modalities. [Respir Care 2015;60(5):724-730. (C) 2015 Daedalus Enterprises]
\end{abstract}

\section{Introduction}

Bronchiectasis is characterized by abnormal, permanent dilation of the bronchi, ${ }^{1}$ which results in chronic cough, mucus production in the airways, and recurrent pulmonary

\footnotetext{
Drs EMC Ramos, D Ramos, and Moreira and Ms Rodrigues are affiliated with the Physical Therapy Department, Presidente Prudente School of Science and Technology, Universidade Estadual Paulist, São Paulo; Drs Macchione, Guimarães, and Saldiva are affiliated with São Paulo University, São Paulo; Dr de Souza is affiliated with the Psychobiology Department, Federal University of São Paulo, São Paulo; and Dr Jardim is affiliated with the Pulmonology Department, Federal University of São Paulo, São Paulo, Brazil.
}

infections. ${ }^{2}$ These respiratory infections can lead to progressive lung damage, ${ }^{3}$ compromising the effectiveness of mucociliary clearance. ${ }^{4}$ Whereas normal bronchial mucus is easily propelled by bronchial cilia, ${ }^{5}$ clearance can be inhibited under abnormal conditions, such as excessive

\footnotetext{
The authors report no conflicts of interest.

Correspondence: EMC Ramos PhD, Physical Therapy Department, Presidente Prudente School of Science and Technology, Universidade Estadual Paulista, São Paulo 19060-900, Brazil. E-mail: ercy@fct.unesp.br.
}

DOI: $10.4187 /$ respcare. 02429 


\section{Effect of Physiotherapy on Mucus Viscoelastic Properties}

mucus production and changes in the viscoelastic properties. $^{6}$

Due to the chronic, progressive nature of bronchiectasis, it is important to establish effective therapeutic strategies ${ }^{3}$ to facilitate mucus clearance and decrease the retention of pulmonary secretions. A number of respiratory physiotherapeutic techniques have been used for this purpose, ${ }^{7}$ such as postural drainage (PD), percussion (PERC), the coughing technique (CT), and other types of cough therapy, such as huffing, employed either alone or in combination. ${ }^{8-20}$ However, the studies cited only report the expectorated volume and did not assess the rheological properties of the mucus. Changes in the rheological properties of mucus may interfere with ciliary clearance and clearance by coughing. ${ }^{21}$ Thus, the analysis of mucus viscosity and elasticity is important, as these elements are crucial to the effective clearance of bronchial secretions.

The aim of the present study was to evaluate the effectiveness of PD, PERC, huffing, and CT and evaluate the quantity (measurement of wet and dry weight) and quality (viscoelastic properties) of the mucus removed using these techniques.

\section{Methods}

\section{Subjects}

Twenty-two subjects ( 6 men and 16 women; mean age: $51.5 \mathrm{y}$; range: $18-76 \mathrm{y}$ ) with non-cystic fibrosis bronchiectasis participated in the study. Cases of cystic fibrosis were diagnosed by the measurement of sodium and chloride in sweat using pilocarpine iontophoresis. All subjects had cylindrical or varicose bronchiectasis, as determined by high-resolution computed tomography. To be included, the subjects had to have been clinically stable with a constant production of mucus and no evidence of respiratory infection in the previous $4 \mathrm{wk}$. Patients with saccular bronchiectasis and those who had changed/interrupted their current medications or began taking new drugs during the trial period were excluded. Nine subjects used bronchodilators during the study period. Ten subjects were former smokers, and 12 were non-smokers.

This study was carried out at the Federal University of São Paulo, Brazil and received approval from the university's human research ethics committee. All subjects signed a statement of informed consent.

\section{Protocol}

The subjects were called to the laboratory for 4 sessions carried out at intervals of $48-72 \mathrm{~h}$. To assess the stability of each subject, expectorated mucus was collected for $24 \mathrm{~h}$ before each experiment, and a urine sample was collected on the morning of the experiment to evaluate urinary den-

\section{QUICK LOOK}

\section{Current knowledge}

Bronchiectasis is characterized by abnormal, permanent dilation of the bronchi, which results in chronic cough, mucus production, and recurrent pulmonary infections. A number of airway clearance techniques have been used to facilitate mucus removal. Most studies cited only report the expectorated volume and do not assess the rheological properties of the mucus.

\section{What this paper contributes to our knowledge}

In a group of subjects with bronchiectasis, postural drainage, percussion, and cough technique and postural drainage with huffing performed similarly regarding the removal of viscoelastic mucus in 2 and 320 -min periods separated by 10 min of rest. Postural drainage, percussion, and cough technique led to the greatest removal of mucus in the shortest time frame, 2 20-min periods separated by $10 \mathrm{~min}$ of rest.

sity and the degree of hydration. The day before the experiment, the subjects were instructed to employ the CT every time they needed to cough and collect the mucus in a receptacle labeled "24-h collector," which was graduated in milliliters. A predetermined amount of mineral oil had been placed in the collector to avoid drying out the mucus and was later subtracted from the total volume in the collector. The receptacle was stored in a cool place until the day of the experiment.

The first day of the experiment was used as the control session and only involved the CT. The subject coughed for $20 \mathrm{~min}$ to expectorate the greatest possible volume of mucus, followed by a 10 -min rest period. The same procedure was repeated twice, for a total of 3 periods $(30 \mathrm{~min}, 60 \mathrm{~min}$, and $90 \mathrm{~min}$ ). The CT performed during the control session differed from the CT of the day before the experiment, as the subjects coughed at predetermined times $(20 \mathrm{~min}$ of coughing followed by a 10 -min rest period over the course of $2 \mathrm{~h}$ ), whereas subjects coughed only when needed on the previous day.

In the 3 subsequent sessions, PD combined with a particular type of cough and/or procedure was performed. The protocol for each session was determined randomly. The following procedures were used: postural drainage + coughing technique $(\mathrm{PD}+\mathrm{CT})$, postural drainage + percussion + coughing technique $(\mathrm{PD}+\mathrm{PERC}+\mathrm{CT})$ and postural drainage + huffing ( $\mathrm{PD}+$ huffing). In a given session, the randomly selected combination of procedures was applied in 320 -min periods interspersed with 10 min of coughing (30 min, $60 \mathrm{~min}$, and $90 \mathrm{~min}$ ). This protocol was chosen to determine whether the typically used 
Effect of Physiotherapy on Mucus Viscoelastic Properties

Table 1. Coughing Maneuvers Performed Throughout the Protocol

\begin{tabular}{|c|c|c|c|c|c|c|c|c|}
\hline $\begin{array}{l}\text { Previous Day } \\
\text { ( } 24 \text { h Before } \\
\text { Each Session) }\end{array}$ & $\begin{array}{l}\text { Session } \\
\text { Type }\end{array}$ & Baseline & $\begin{array}{l}\text { Rest } \\
\text { Period }\end{array}$ & At $30 \mathrm{~min}$ & $\begin{array}{c}\text { Rest } \\
\text { Period }\end{array}$ & At $60 \mathrm{~min}$ & $\begin{array}{l}\text { Rest } \\
\text { Period }\end{array}$ & At $90 \mathrm{~min}$ \\
\hline $\mathrm{CT}$ & $\mathrm{CT}$ & $\mathrm{CT}$ & $10 \mathrm{~min}$ & $\mathrm{CT}$ & $10 \mathrm{~min}$ & CT & $10 \mathrm{~min}$ & $\mathrm{CT}$ \\
\hline CT & $\mathrm{PD}+\mathrm{CT}$ & $\mathrm{CT}$ & $10 \mathrm{~min}$ & $\mathrm{CT}$ & $10 \min$ & CT & $10 \mathrm{~min}$ & $\mathrm{CT}$ \\
\hline $\mathrm{CT}$ & $\mathrm{PD}+\mathrm{PERC}+\mathrm{CT}$ & $\mathrm{CT}$ & $10 \mathrm{~min}$ & $\mathrm{CT}$ & $10 \mathrm{~min}$ & $\mathrm{CT}$ & $10 \mathrm{~min}$ & $\mathrm{CT}$ \\
\hline $\mathrm{CT}$ & PD+huffing & Huffing & $10 \mathrm{~min}$ & Huffing & $10 \mathrm{~min}$ & Huffing & $10 \mathrm{~min}$ & Huffing \\
\hline $\begin{array}{l}\mathrm{CT}=\text { cough techniqu } \\
\mathrm{PD}=\text { postural draina } \\
\mathrm{PERC}=\text { percussion }\end{array}$ & & & & & & & & \\
\hline
\end{tabular}

20-min duration would be sufficient to remove mucus with changed viscoelastic properties or whether there was a need to increase the application time of the technique, respecting a period of $10 \mathrm{~min}$ for the appropriate handling of expectorated sputum.

On each day of the experiment $(\mathrm{CT}, \mathrm{PD}+\mathrm{CT}$, $\mathrm{PD}+\mathrm{PERC}+\mathrm{CT}$, and PD + huffing), subjects were initially asked to cough for $20 \mathrm{~min}$ using the $\mathrm{CT}$ to expectorate the greatest possible volume of mucus and allow the determination of the volume and rheological properties of the sputum in the baseline period. This protocol was performed to standardize the experiment so that all subjects began the experiment with the same bronchial hygiene before each session. After each 20-min period of the applied technique (30 $\mathrm{min}, 60 \mathrm{~min}$, and $90 \mathrm{~min}$ ), the subject was asked to cough to eliminate all the mucus produced. Depending on the procedure used, the mucus could be produced by the CT or huffing. Table 1 demonstrates the study design with regard to the session type as well as the type of cough performed in each moment after session.

The lateral decubitus position was selected for PD based on the lobe with the greatest adventitious breath sounds. The selection of right or left lateral decubitus was based on auscultation of the lung segments, which was performed with the subject in the sitting position. The lung segment with the greatest adventitious breath sounds was placed in the upward lateral position. The technique was applied for $20 \mathrm{~min}$ and the subject was then instructed to cough and expectorate. After $10 \mathrm{~min}$ of rest, the therapist auscultated again and determined the position for the second period. Thus, the position could vary with every 20 -min period based on the results of the auscultation.

Percussion was performed at a rate of approximately 500 beats/min, alternating both hands. For CT, the subject was instructed to breathe slowly and deeply using the diaphragm and intercostal muscles, followed by a short apnea and opening the glottis with an abrupt expulsion of lung volume. With the huffing technique, the subject inspired an average lung volume, followed by 2 expiratory efforts (huffs).
The subject was instructed before the procedure to cough and expectorate the mucus only after the end of each period. Therefore, one aliquot was obtained for each period. The expectorated mucus samples were stored in Eppendorf tubes under mineral oil to minimize the effects of dehydration during the cooling and storage period. ${ }^{22,23} \mathrm{Be}-$ fore analysis, the mineral oil was removed through immersion in petroleum ether. The samples were stored at $-20^{\circ} \mathrm{C} .{ }^{22}$ The collected mucus was stored within $5 \mathrm{~min}$ to minimize contact with the air and avoid dehydration, which would alter the physical properties. ${ }^{24}$

For the qualitative assessment of the expectorated mucus, a sample from each collection period was placed on a sterile blade and weighed on an analytical balance. The blade was then placed in a microwave oven at full power for $20 \mathrm{~min}$ and re-weighed. The wet and dry mucus weight was obtained by subtracting the weight of the blade alone from the weight of the blade with the mucus before and after drying. The ratio of dry to wet weight was computed and expressed as percentage of solids.

\section{Viscosity and Elasticity}

A dual chamber capillary viscometer was used to measure the viscosity and elasticity of the bronchial mucus. ${ }^{25-27}$ The mucus sample filled the steel capillary to $0.2 \mathrm{~cm}$ from the distal edge. The sample was subjected to negative pressure for displacement, and the distance and time were recorded. After displacement had ceased, the pressure was removed and the recoil of the sample within the glass capillary was recorded. The Poiseuille equation was used to measure the viscosity (in poise) and elasticity (in dynes/cm) of the mucus:

$$
\eta=\left(\pi \times \mathrm{r}^{4} \times \Delta \mathrm{P}\right) / 8 \times 1 \times \mathrm{Q}
$$

in which $\eta=$ viscosity, $\mathrm{r}=$ tube radius (in $\mathrm{cm}$ ), $\Delta \mathrm{P}=$ pressure of the sample through the tube (in $\mathrm{cm}_{2} \mathrm{O}$, which 


\section{Effect of Physiotherapy on Mucus Viscoelastic Properties}

was converted to dynes $\left./ \mathrm{cm}^{2}\right), 1=$ tube length (in $\mathrm{cm}$ ), and $\mathrm{Q}=$ volumetric flow

Using the viscosity value, elasticity (dynes/cm), or elastic modulus, was obtained by the application of Hooke's law:

$$
\mathrm{F}=\mathrm{k} \times \Delta \mathrm{l}
$$

in which $\mathrm{F}=$ the pressure applied to the system $(\mathrm{F}=\mathrm{a} \times \mathrm{P}$, in which $\mathrm{a}=$ area (in the present study: $\pi \times \mathrm{r}^{2}$, with $\pi=3.1416$ and $\mathrm{r}=0.055 \mathrm{~cm}$ ) and $\mathrm{P}=$ pressure in $\mathrm{cm}$ $\mathrm{H}_{2} \mathrm{O}$, which was converted to dyne $/ \mathrm{cm}^{2}, \Delta \mathrm{l}=$ recoil of the fluid through the capillary (in $\mathrm{cm}$ ), and $\mathrm{k}=$ constant of the elastic material being measured. ${ }^{11}$ During the viscoelastic measurements, the laboratory room temperature was maintained at $26^{\circ} \mathrm{C}$.

\section{Statistical Analysis}

Statistical analysis was performed with SPSS 17.0 (SPSS, Chicago, Illinois). All data were expressed as mean \pm SD values. A repeated-measures general linear model was used, employing a 4 (session) $\times 4$ (period) within-subjects model. This procedure considered sessions $(\mathrm{CT}, \mathrm{PD}+\mathrm{CT}$, $\mathrm{PD}+\mathrm{PERC}+\mathrm{CT}$, and $\mathrm{PD}+$ huffing) and periods (baseline, 30,60 , and $90 \mathrm{~min}$ ), as well as their interactions in a single model. For the period-session interaction, changes in dry weight/wet weight ratio, viscosity, and elasticity among periods in each session were determined (Figs. 1-3). The Bonferroni test was used for post hoc analysis. Statistical significance was set to $5 \%(P<.05)$.

\section{Results}

The stability of each subject was assessed by the quantification of expectorated mucus on the day before each experiment and the measurement of density of the urine sample on the morning of the experiment. The subjects were clinically stable during the course of the study, as no significant differences in mucus volume $(\mathrm{mL})(\mathrm{CT}=$ $24.9 \pm 10.7 ; \mathrm{PD}+\mathrm{CT}=25.79 \pm 8.6 ; \mathrm{PD}+\mathrm{PERC}+\mathrm{CT}=$ $26.6 \pm 9.7$ and $\mathrm{PD}+$ huffing $=27.4 \pm 8.6, P=.62$ ) or urinary density $\left(\mathrm{kg} / \mathrm{m}^{3}\right)(\mathrm{CT}=1,013.8 \pm 4.6 ; \mathrm{PD}+\mathrm{CT}=$ $1,012.9 \pm 6.1 ; \mathrm{PD}+\mathrm{PERC}+\mathrm{CT}=1,012.9 \pm 4.5$ and $\mathrm{PD}+$ huffing $=1,012.9 \pm 4.8, P=.86$ ) were demonstrated before performing the different treatments.

Figure 1 illustrates the dry weight/wet weight ratio for the sessions and evaluation periods. The percentage of solids content at $60 \mathrm{~min}$ was greater following PD + PERC $+\mathrm{CT}$ compared to CT (session effect $P=.01$ ). At $90 \mathrm{~min}$, a significant increase was found in the percentage of solids content removed following $\mathrm{PD}+\mathrm{PERC}+\mathrm{CT}$ and $\mathrm{PD}+$ huffing compared to $\mathrm{CT}$ (session effect $P=.007$ and

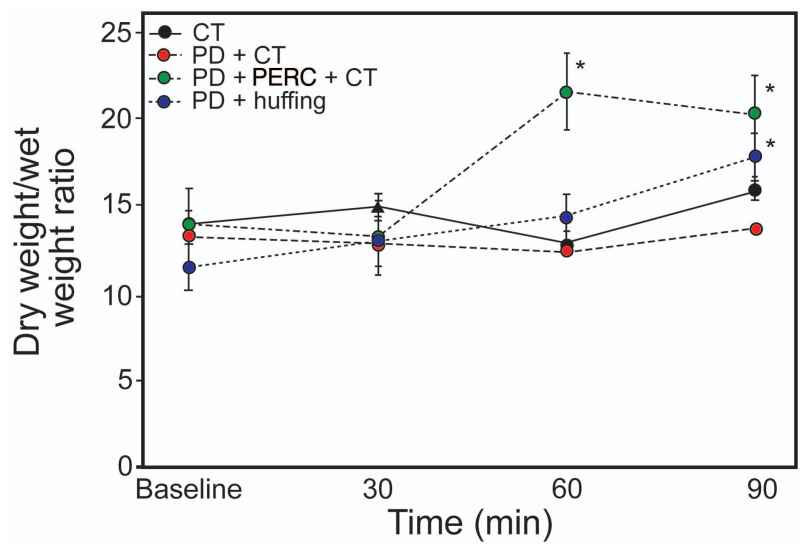

Fig. 1. Results of interactions among sessions and periods for dry weight/wet weight ratio. The symbols represent statistically significant differences between sessions and periods, according to Bonferroni post hoc analysis $(P<.05)$. Data are shown as mean $\pm \mathrm{SD}$. * = statistically significant vs $\mathrm{CT}$ and $\mathrm{PD}+\mathrm{CT}$.

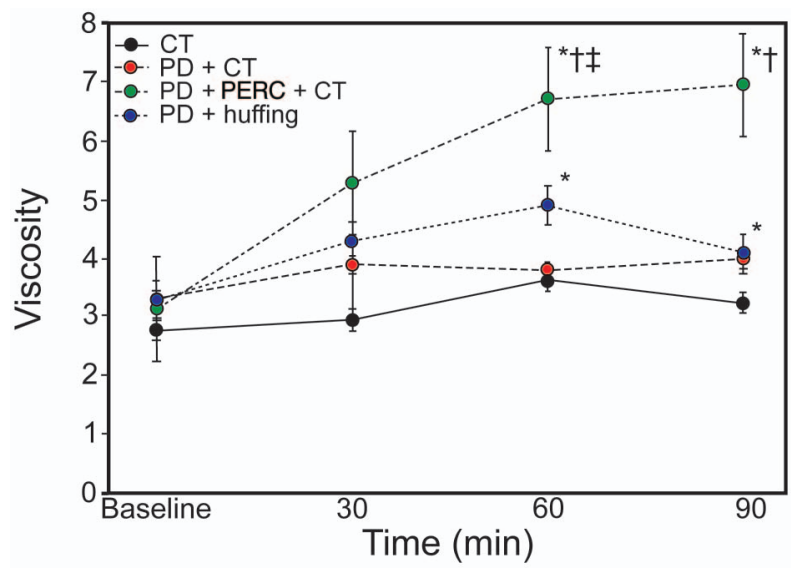

Fig. 2. Results of interactions among sessions and periods for mucus viscosity. The symbols represent statistically significant differences between sessions and periods, according to Bonferroni post hoc analysis $(P<.05)$. Data are shown as mean \pm SD. * = statistically significant compared with CT; $\dagger=$ statistically significant compared with $\mathrm{PD}+\mathrm{CT} ; \ddagger=$ statistically significant compared with baseline.

$P=.03$, respectively) and PD $+\mathrm{CT}$ (session effect $P=.01$ and $P=.04$, respectively). No significant differences in the dry weight/wet weight ratios were found among the periods and session-period interaction $(P>.05$, for all).

Figure 2 illustrates the bronchial mucus viscosity values for the sessions and periods. PD $+\mathrm{PERC}+\mathrm{CT}$ led to a greater removal of viscous mucus at 60 and $90 \mathrm{~min}$ compared to baseline (period effect $P=.01$ and $P=.001$, respectively) and CT (session effect $P=.02$ and $P=.001$, at 60 and 90 min respectively) and $\mathrm{PD}+\mathrm{CT}$ (session effect $P=.04)$. Moreover, a session-period interaction was found, as the viscosity of the mucus at $60 \mathrm{~min}$ was greater following PD + PERC $+\mathrm{CT}$ (interaction effect $P=.02$ ) 


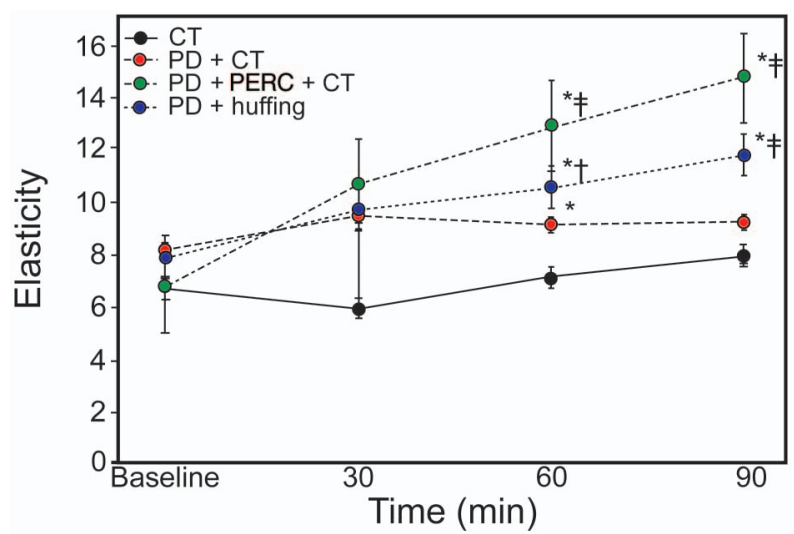

Fig. 3. Results of interactions among sessions and periods for mucus elasticity. The symbols represent statistically significant differences between sessions and periods, according to Bonferroni post hoc analysis $(P<.05)$. Data are shown as mean \pm SD.

* = statistically significant compared with $\mathrm{CT} ; \dagger=$ statistically significant compared with $\mathrm{PD}+\mathrm{CT} ; \ddagger=$ statistically significant compared with baseline.

and PD+huffing (interaction effect $P=.002$ ) in comparison with CT. These differences were also found at $90 \mathrm{~min}$ (interaction effect $P=.02$ and $P=.01$, respectively).

Figure 3 illustrates the bronchial mucus elasticity values for each period and session. $\mathrm{PD}+\mathrm{PERC}+\mathrm{CT}$ led to a greater removal of elastic mucus at $60 \mathrm{~min}($ period effect $P=.001)$, whereas PD+huffing led to a greater removal of elastic mucus only at $90 \mathrm{~min}$ (period effect $P=.007$ ) in comparison with baseline. An interaction effect was found, as all techniques led to a greater removal of elastic mucus in comparison with $\mathrm{CT}$ at $60 \mathrm{~min}$ (interaction effect for $\mathrm{PD}+\mathrm{CT}, P=.001 ; \mathrm{PD}+\mathrm{PERC}+\mathrm{CT}, P<.001$; $\mathrm{PD}+$ huffing, $P<.001$ ), but only $\mathrm{PD}+\mathrm{PERC}+\mathrm{CT}$ and PD +huffing led to a greater removal of elastic mucus than $\mathrm{CT}$ at $90 \mathrm{~min}$ (interaction effect $P<.001$ and $P=.005$, respectively).

\section{Discussion}

A number of studies have investigated the effectiveness of respiratory physiotherapeutic techniques for the removal of mucus. ${ }^{8-20}$ However, most studies evaluated efficacy based on spirometry, ${ }^{8,28,29}$ the inhalation of a radioaerosol $^{30}$ or the volume of expectorated secretion. ${ }^{10,12,15,18,19,31,32}$ Thus, the aim of the present study was to examine the viscoelasticity of mucus removed following PD, percussion, and huffing in subjects with cylindrical and/or varicose bronchiectasis.

The present findings demonstrate that PD $+\mathrm{PERC}+\mathrm{CT}$ and $\mathrm{PD}+$ huffing led to more efficient removal of mucus with the worst rheological properties. However, satisfactory effects were found only when these methods were applied for 220 -min periods separated by $10 \mathrm{~min}$ of rest.
The relative amount of mucus expectorated during each session was assessed by the dry weight/wet weight ratio, and bronchial mucus viscoelasticity was analyzed using a dual chamber capillary viscometer due to its easy preparation and handling. The measurements were obtained quickly, and this device is simpler and more practical than the magnetic micro-rheometer device used in most studies to determine the rheological properties of bronchial mucus. Saliva was easily separated from mucus ${ }^{33}$ due to its more liquid and foamy characteristics. ${ }^{34}$ The baseline measurements of viscoelasticity and mucus quantity were similar in the 4 sessions of the experiment, demonstrating that the subjects remained stable and also that the effect of duration following application of the respiratory therapy techniques was less than $48 \mathrm{~h}$. Thus, the treatments had only an acute effect on the quantity and quality of the bronchial mucus expectorated.

The evaluation of the dry weight/wet weight ratio indicates the true quantity of mucus expectorated. As shown in Table 1, PD + PERC $+\mathrm{CT}$ led to the removal of the greatest amount of mucus in the shortest period $(60 \mathrm{~min})$ followed by PD+huffing $(90 \mathrm{~min}$ ) in comparison with $\mathrm{CT}$ and $\mathrm{PD}+\mathrm{CT}$. These results are in agreement with previous studies, which found that the combination of percussion and PD was more effective in terms of the volume of expectorated mucus than coughing alone in subjects with stable chronic bronchitis ${ }^{35}$ and that forced exhalation (such as huffing) combined with PD was more effective than CT with regard to sputum production and radioisotope clearance. $^{20}$

In the present study, the physicochemical properties of expectorated mucus were analyzed to determine differences in the effectiveness of physiotherapy techniques. The results show that $\mathrm{PD}+\mathrm{PERC}+\mathrm{CT}$ and $\mathrm{PD}+$ huffing led to the greatest removal of viscous mucus at both $60 \mathrm{~min}$ and 90 min (see Fig. 2). In subjects with clinically stable chronic air-flow obstruction, PD and coughing with or without PERC have been found to improve mucociliary clearance in a 20 -min session. ${ }^{18}$ In the present study, PD + PERC led to the removal of more viscous mucus, but at least 2 20-min periods were required (see Fig. 2). The difference in duration may be explained by differences in the method used to assess mucociliary transport and the combination of breathing exercises with these techniques in the study cited.

Regarding mucus elasticity, all techniques led to the removal of secretions that were more elastic (ie, had a smaller elastic modulus) than that obtained by $\mathrm{CT}$ in 60 min (see Table 1), but only PD + PERC + CT and PD + huffing continued to achieve significant differences at $90 \mathrm{~min}$, demonstrating that these techniques were able to move mucus with a higher elastic modulus throughout the session. Huffing produces higher expiratory flow ${ }^{36}$ by dynamic compression and collapse of the airways 


\section{Effect of Physiotherapy on Mucus Viscoelastic Properties}

downstream of the equal pressure point, which helps to clear and mobilize secretions. In addition, the results of the present study showed that percussion tends to be a frequency- and time-dependent technique because better rheological mucus properties were obtained with more sessions or longer duration of each session. So, these situations may have contributed to removing mucus with greater elasticity.

$\mathrm{PD}+\mathrm{PERC}+\mathrm{CT}$ and $\mathrm{PD}+$ huffing proved to be the most effective techniques for the removal of mucus with the worst rheological profile when applied for 220 -min periods. This indicates that PD+PERC $+\mathrm{CT}$ and PD+huffing removed secretions that would otherwise be difficult to clear, as more elastic and viscous mucus exhibits greater impedance, which hinders its removal. ${ }^{37,38} \mathrm{~A}$ number of in vitro studies on human bronchial mucus have demonstrated an inverse relationship between mucociliary clearance and viscoelastic properties. ${ }^{23,24,38-41}$ So, when it is not possible to execute the percussion due to contraindications or a lack of patient tolerance, the huffing technique may be considered in daily practice. Furthermore, it is important to note that performing any type of airway clearance technique for the usual duration of 20 min may not differ from using CT alone this particular patient population and the therapist may need more time (at least 220 -min periods) to obtain a significant effect on mucus viscoelasticity (see Figs. 2 and 3).

It is difficult to compare the present findings with data in the literature due to the small number of studies that have evaluated the effectiveness of the techniques employed herein with regard to the rheological properties of mucus. App et $\mathrm{al}^{42}$ analyzed the effects of using flutter and autogenic drainage on the rheological properties of mucus removed from patients with cystic fibrosis and concluded that the mucus viscoelasticity was significantly lower after treatment with flutter, suggesting that this technique changes mucus properties. Some airway clearance techniques have important physiological differences on rheological properties of mucus. The application of manual techniques, such as percussion, remove secretions without changes in mucus viscoelasticity; however, devices such as flutter that offer a combination of positive airway pressure and oscillation of air within airways during expiration change mucus properties, reducing the viscoelasticity of sputum. ${ }^{43}$

Therefore, it is important to know physiological principles of physiotherapeutic techniques and to determine the effects of these techniques on the rheological properties of the mucus, which is a factor for the effective clearance of bronchial secretions. ${ }^{44}$ Mucus with a higher degree of viscoelasticity is more difficult to remove spontaneously, and, logically, more mucus in the bronchi may lead to the proliferation of bacteria, making the patient more susceptible to respiratory infection and recurrent exacerbations.
Despite the lack of scientific evidence, the identification and application of techniques that are effective in leading to the removal of mucus with high viscoelasticity may contribute to reductions in exacerbations and the frequency of hospital admissions, thereby leading to an improvement in quality of life. There is a lack of randomized control trials that investigate the effect of bronchopulmonary hygiene techniques on these clinical outcomes. Such assessments are important and should be considered in further research.

There are several study limitations that warrant consideration in the interpretation of our results. First, the sample size was small, but subjects participated in 4 separate sessions, yielding a large number of therapeutic observations. Also, there was no separate control group; however, subjects served as their own control, and procedures were used to measure baseline stability and variances. These control sessions were conducted prior to research initiation and the start of each protocol session, and analysis demonstrated no statistically significant difference in subject baseline sputum volume at any time period. In addition, applicability of these results to actual clinical practice may be limited by provider time constraints to perform therapy for the duration used in our study. Reproducibility of our results may also depend on provider technique in performing the various airway-clearance modalities.

\section{Conclusions}

The present findings demonstrate that $\mathrm{PD}+\mathrm{PERC}+\mathrm{CT}$ and PD+huffing achieve similar effects regarding the removal of more viscous and more elastic mucus in 220 -min periods compared to $\mathrm{CT}$ alone. Moreover, $\mathrm{PD}+\mathrm{PERC}+\mathrm{CT}$ led to the removal of the greatest amount of secretions in the shortest period $(60 \mathrm{~min})$.

\section{REFERENCES}

1. King PT. The pathophysiology of bronchiectasis. Int J Chron Obstruct Pulmon Dis 2009;4:411-419.

2. Morrissey BM. Pathogenesis of bronchiectasis. Clin Chest Med 2007; 28(2):289-296.

3. Vendrell M, de Gracia J, Olveira C, Martínez MA, Girón R, Máiz L, et al. [Diagnosis and treatment of bronchiectasis. Spanish Society of Pneumology and Thoracic Surgery] Arch Bronconeumol. 2008; 44(11):629-640.

4. Shibuya Y, Wills PJ, Cole PJ. Effect of osmolality on mucociliary transportability and rheology of cystic fibrosis and bronchiectasis sputum. Respirology 2003;8(2):181-185.

5. Foster WM. Mucociliary transport and cough in humans. Pulm Pharmacol Ther 2002;15(3):277-282.

6. Martins ALP JM, Costa D. Estudo das propriedades reológicas do muco brônquico de pacientes submetidos a técnicas de fisioterapia respiratória. Rev Bras Fisioter 2005;9(1):33-39.

7. McCool FD, Rosen MJ. Nonpharmacologic airway clearance therapies: ACCP evidence-based clinical practice guidelines. Chest 2006; 129(1 Suppl):250S-259S. 


\section{Effect of Physiotherapy on Mucus Viscoelastic Properties}

8. Anthonisen P, Riis P, Sogaard-Andersen T. The value of lung physiotherapy in the treatment of acute exacerbations in chronic bronchitis. Acta Med Scand 1964;175:715-719.

9. Cherniack RM. Physical therapy. Am Rev Respir Dis 1980;122(5 Pt 2):25-27.

10. Oldenburg FA Jr, Dolovich MB, Montgomery JM, Newhouse MT. Effects of postural drainage, exercise, and cough on mucus clearance in chronic bronchitis. Am Rev Respir Dis 1979;120(4):739-745.

11. Pryor JA, Webber BA, Hodson ME, Batten JC. Evaluation of the forced expiration technique as an adjunct to postural drainage in treatment of cystic fibrosis. BMJ 1979;2(6187):417-418.

12. Mortensen J, Falk M, Groth S, Jensen C. The effects of postural drainage and positive expiratory pressure physiotherapy on tracheobronchial clearance in cystic fibrosis. Chest 1991;100(5):1350-1357.

13. Verboon JM, Bakker W, Sterk PJ. The value of the forced expiration technique with and without postural drainage in adults with cystic fibrosis. Eur J Respir Dis 1986;69(3):169-174.

14. de Boeck C, Zinman R. Cough versus chest physiotherapy: a comparison of the acute effects on pulmonary function in patients with cystic fibrosis. Am Rev Respir Dis 1984;129(1):182-184.

15. van Hengstum M, Festen J, Beurskens C, Hankel M, Beekman F, Corstens $\mathrm{F}$. Conventional physiotherapy and forced expiration manoeuvres have similar effects on tracheobronchial clearance. Eur Respir J 1988;1(8):758-761.

16. Holland AE, Button BM. Is there a role for airway clearance techniques in chronic obstructive pulmonary disease? Chron Respir Dis 2006;3(2):83-91.

17. Mazzocco MC, Owens GR, Kirilloff LH, Rogers RM. Chest percussion and postural drainage in patients with bronchiectasis. Chest 1985;88(3):360-363.

18. van der Schans CP, Piers DA, Postma DS. Effect of manual percussion on tracheobronchial clearance in patients with chronic airflow obstruction and excessive tracheobronchial secretion. Thorax 1986; 41(6):448-452.

19. Rossman CM, Waldes R, Sampson D, Newhouse MT. Effect of chest physiotherapy on the removal of mucus in patients with cystic fibrosis. Am Rev Respir Dis 1982;126(1):131-135.

20. Sutton PP, Parker RA, Webber BA, Newman SP, Garland N, LopezVidriero MT, et al. Assessment of the forced expiration technique, postural drainage and directed coughing in chest physiotherapy. Eur J Respir Dis 1983;64(1):62-68.

21. Zahm JM, Pierrot D, Vaquez-Girod S, Duvivier C, King M, Puchelle E. The role of mucus sol phase in clearance by simulated cough. Biorheology 1989;26(4):747-752.

22. Gastaldi AC, Jardim JR, King M. The influence of temperature and length of time of storage of frog mucus samples. Biorheology 2000; 37(3):203-211.

23. Dulfano MJ, Adler KB. Physical properties of sputum. VII. Rheologic properties and mucociliary transport. Am Rev Respir Dis 1975; 112(3):341-347

24. King M, Agarwal M, Shukla JB. A planar model for mucociliary transport: effect of mucus viscoelasticity. Biorheology 1993;30(1): 49-61.

25. Barnett B, Dulfano MJ, Philippoff W, Han CD. A method for determining the viscoelastic properties of bioogical fluids. Biorheology 1970;7(1):55-67.

26. Kim CS, Berkley BB, Abraham WM, Wanner A. A micro double capillary method for rheologic measurements of lower airway secretions. Bull Eur Physiopathol Respir 1982;18(6):915-927.
27. Kim CS. Capillary type viscometer. In: Braga PCAL, editor. Methods in bronchial mucology. New York, NY: Raven Press; 1988:93103

28. Campbell AH, O'Connell JM, Wilson F. The effect of chest physiotherapy upon the FEV1 in chronic bronchitis. Med J Aust 1975; 1(2):33-35.

29. Giles DR, Wagener JS, Accurso FJ, Butler-Simon N. Short-term effects of postural drainage with clapping vs autogenic drainage on oxygen saturation and sputum recovery in patients with cystic fibrosis. Chest 1995;108(4):952-954.

30. Newman SP, Agnew JE, Pavia D, Clarke SW. Inhaled aerosols: lung deposition and clinical applications. Clin Phys Physiol Meas 1982; 3(1): $1-20$

31. Marks JH, Hare KL, Saunders RA, Homnick DN. Pulmonary function and sputum production in patients with cystic fibrosis: a pilot study comparing the PercussiveTech HF device and standard chest physiotherapy. Chest 2004;125(4):1507-1511.

32. Placidi G, Cornacchia M, Polese G, Zanolla L, Assael BM, Braggion C. Chest physiotherapy with positive airway pressure: a pilot study of short-term effects on sputum clearance in patients with cystic fibrosis and severe airway obstruction. Respir Care 2006;51(10): 1145-1153.

33. Proctor DF AE, Reasor MJ, Bucklen KR. A method for collecting normal respiratory mucus. In: Puchelle EM, editor. Rheology of bronchial secretions and respiratory functions. Paris, France; 1973: 351-356.

34. Puchelle E, Tournier JM, Zahm JM, Sadoul P. Rheology of sputum collected by a simple technique limiting salivary contamination. J Lab Clin Med 1984;103(3):347-353.

35. May DB, Munt PW. Physiologic effects of chest percussion and postural drainage in patients with stable chronic bronchitis. Chest 1979;75(1):29-32.

36. Lapin CD. Airway physiology, autogenic drainage, and active cycle of breathing. Respir Care 2002;47(7):778-785

37. Zanchet RC, Feijó G, Gastaldi AC, Jardim JR. Physicochemical properties of human tracheobronchial sputum maintained at room temperature. J Bras Pneumol 2007;33(1):57-61.

38. Macchione M, King M, Lorenzi-Filho G, Guimarães ET, Zin WA, Böhm GM, Saldiva PH. Rheological determinants of mucociliary transport in the nose of the rat. Respir Physiol 1995;99(1):165-172.

39. King M. Viscoelastic properties of airway mucus. Fed Proc 1980; 39(13):3080-3085.

40. King M, Gilboa A, Meyer FA, Silberberg A. On the transport of mucus and its rheologic simulants in ciliated systems. Am Rev Respir Dis 1974;110(6):740-745.

41. Puchelle E, Zahm JM, Quemada D. Rheological properties controlling mucociliary frequency and respiratory mucus transport. Biorheology 1987;24(6):557-563.

42. App EM, Kieselmann R, Reinhardt D, Lindemann H, Dasgupta B, King M, Brand P. Sputum rheology changes in cystic fibrosis lung disease following two different types of physiotherapy: flutter vs autogenic drainage. Chest 1998;114(1):171-177.

43. Rogers D. DIJM. Physiological principles of airway clearance techniques used in the physiotherapy management of cystic fibrosis. Curr Paediatr 2005;15(1):233-238.

44. Trindade SH, de Mello JF Jr, Mion Ode G, Lorenzi-Filho G, Macchione M, Guimarães ET, Saldiva PH. Methods for studying mucociliary transport. Braz J Otorhinolaryngol 2007;73(5):704-712. 